\title{
On the properties of GaP supersaturated with $\mathrm{Ti}$
}

\author{
J. Olea ${ }^{1}$, S. Algaidy ${ }^{1}$, A. del Prado ${ }^{1}$, E. García-Hemme ${ }^{1}$, R. García-Hernansanz ${ }^{1}$, D. Montero ${ }^{1}$, D. \\ Caudevilla ${ }^{1}$, G. González-Díaz ${ }^{1}$, E. Soria ${ }^{2,3}$, J. Gonzalo ${ }^{2}$ \\ ${ }^{1}$ Departamento de Estructura de la Materia, Física Térmica y Electrónica, Facultad de Ciencias Físicas, Universidad Complutense de Madrid, 28040 Madrid, Spain. \\ ${ }^{2}$ Laser Processing Group, Instituto de Optica, IO-CSIC, Serrano 121, 28006 Madrid, Spain. \\ ${ }^{3}$ SAFTRA Photonics, Jesenná 5, 04001 Košice, Slovakia.
}

\begin{abstract}
We have fabricated GaP supersaturated with Ti by means of ion implantation and pulsed-laser melting to obtain an intermediate band material with applications in photovoltaics. This material has a strong sheet photoconductance at energies below the bandgap of GaP and it seems to be passivated by a Ga defective GaPO oxide layer during the laser process. Passivation is consistently analyzed by sheet photoconductance and photoluminescence measurements. We report on the structural quality of the resulting layers and analyze the energy of the new optical transitions measured on GaP:Ti. A collapse found in the sheet photoconductance spectra of GaP:Ti samples fabricated on undoped substrates is explained by the negative photoconductivity phenomenon.
\end{abstract}

Index Terms - Gallium compounds, Ion implantation, Laser applications, Photovoltaic cells, Titanium.

\section{INTRODUCTION}

G ALLIUM phosphide research has received renewed interest in the field of photovoltaics due to its potential for $\mathrm{GaP} / \mathrm{Si}$ tandem devices [1][2] and as a candidate as top-cell in 5J multijunction solar cells [3]. Moreover, Intermediate Band (IB) solar cells based on $\mathrm{GaP}$ could theoretically reach efficiencies close to $60 \%$ [4][5]. In order to form an IB in GaP, one could introduce an extremely high concentration of a deep center (i.e. Ti), above the Luque's limit, originating the overlapping of the deep levels and yielding an insulator-to-metal transition [6]. Wide bandgap semiconductors are advantageous in the field of IB materials, since it is easier to form an IB not overlapped with the Conduction Band (CB) or the Valence Band (VB). The overlapping of the IB would reduce the effective open circuit voltage of the device and the effective spectral range [7]. Additionally, very recently a theoretical study highlighted also the interest of Ti doped $\mathrm{GaP}$ in the field of spintronics [8].

Recently, $\mathrm{GaP}$ supersaturated with $\mathrm{Ti}$ was fabricated by $\mathrm{Ti}$ ion implantation and Pulsed Laser Melting (PLM) [4]. The characterization of this material yielded a strong sheet photoconductance at energies below the GaP bandgap, which was predicted theoretically [9][10]. This photoconductance had a front at about $1.1 \mathrm{eV}$ at room temperature. This enhanced photoconductance could increase the efficiency of a $\mathrm{GaP}$ based solar cell. Although other methods are being explored, such as electron-beam pulse annealing [11], PLM seems to be an interesting fabrication process for III-V semiconductors [12]. This technique is able to melt and recrystallize a layer implanted with an extremely high dose, producing a high quality supersaturated lattice, and even overcoming the solid solubility limit of the impurity in GaP. So far, most of the literature referred to GaP supersaturated by PLM has been devoted to GaP:Mn and its magnetic properties [13][14] and therefore there is a lack of research using other impurities or looking for other potential applications.

In [4] we concluded that apart from the new photoconductance range added, a passivation effect could be acting as a result of the PLM process, but more experiments were necessary to give definitive conclusions. Also, an abrupt collapse of the photoconductance at about $550 \mathrm{~nm}$ was presented, and being such an odd result, more insights might be necessary to explain its origin. Finally, even though the PLM process window was clearly defined to obtain a high quality material, it was done from the electronic transport properties point of view, and therefore a dedicated structural analysis of the fabricated material is mandatory. Moreover, the electronic transport properties (conduction type, carrier mobility and concentration) of the GaP:Ti material have not been analyzed yet. The use of a highly conductive substrate hid these properties, since the substrate conduction dominated in the experiment.

The passivation of the GaP surface is extremely important for optoelectronic devices based on this semiconductor. Due to this problem, the maximum efficiency of practical $\mathrm{GaP}$ solar

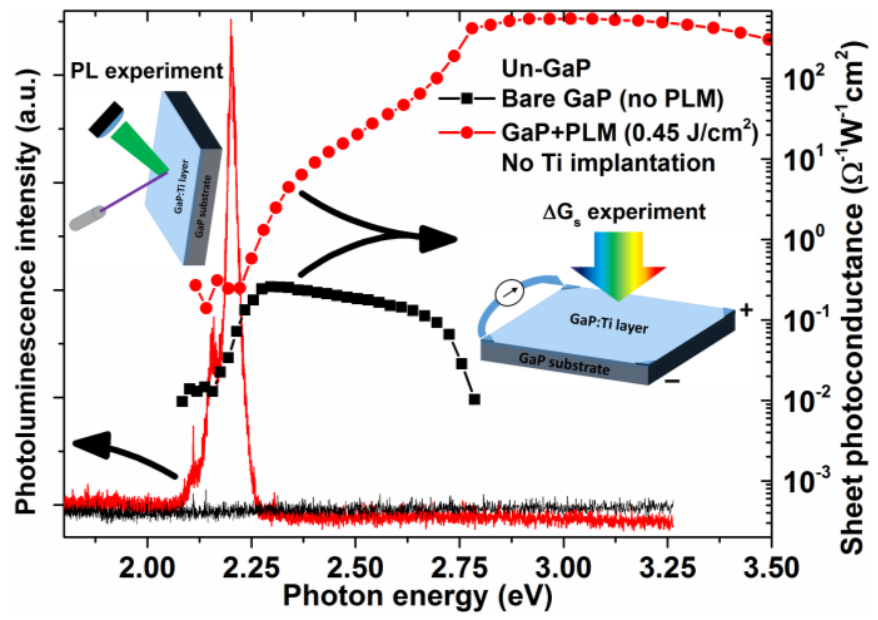

Fig. 1. Room temperature sheet photoconductance (symbols and lines) and 10 $\mathrm{K}$ photoluminescence (lines) spectra of two unimplanted Un-GaP samples as a function of the photon energy. The as-grown sample is depicted in black while the PLM processed sample is depicted in red. The figure shows also two sketches of the sheet photoconductance (right) and photoluminescence (left) experiments. 
cells is below 5\% [15], even though the theoretical ShockleyQueisser limit for GaP is around 20\% [16]. Thus, the fabrication of a $\mathrm{GaP}: \mathrm{Ti}$ IB material with improved photoresponse and the passivation of its surface in the same fabrication process could be highly convenient.

In this work we advance in the understanding of the properties of GaP:Ti fabricated by ion implantation and PLM, analyzing the electronic, optoelectronic and structural properties.

\section{EXPERIMENTAL}

Two different GaP substrates were used in this work, both of them single side polished and grown in the (100) direction. One of them was an n-type undoped GaP substrate and the other a semi-insulating GaP substrate (Un-GaP and SI-GaP, respectively). A summary of the properties of both substrates at room temperature is shown in Table 1. Both substrate types were implanted with ${ }^{48} \mathrm{Ti}^{+}$at $32 \mathrm{keV}$ in a Varian $\mathrm{CF} 3000$ ion implanter refurbished by IBS France, using $\mathrm{TiCl}_{3}$ as a precursor. Two Ti doses were implanted: $8 \times 10^{14} \mathrm{~cm}^{-2}$ and $2 \times 10^{15} \mathrm{~cm}^{-2}$, both resulting in Ti concentration overcoming the Luque's limit [4][6]. Implantation processes were conducted on the polished surface at $7^{\circ}$ tilt in order to reduce channeling.

\begin{tabular}{|c|c|c|}
\hline Type & Un-GaP & SI-GaP \\
\hline $\begin{array}{c}\text { Electron } \\
\text { concentration }\left(\mathbf{c m}^{-3}\right)\end{array}$ & $2-8 \times 10^{16}$ & $0.63-1.87 \times 10^{8}$ \\
\hline Mobility $\left(\mathbf{c m}^{2} / \mathbf{V s}\right)$ & 120 & $168-201$ \\
\hline Resistivity $(\mathbf{\Omega c m})$ & $0.65-2.6$ & $1.99-4.97 \times 10^{8}$ \\
\hline Thickness $(\boldsymbol{\mu m})$ & 500 & 450 \\
\hline
\end{tabular}

Table 1: Electronic transport properties of the GaP substrates used in this work.

In order to recover the crystal quality of the implanted layer and to form a supersaturated GaP:Ti material, implanted samples were PLM processed with an ArF excimer laser $(\lambda=$ $193 \mathrm{~nm}, 20 \mathrm{~ns}$ full width half maximum). PLM was conducted in air with a $0.95 \times 0.95 \mathrm{~mm}^{2}$ beam size at the irradiation plane, stepping just one pulse over the whole surface with an overlapping of $5 \mu \mathrm{m}$ horizontally and $10 \mu \mathrm{m}$ vertically. The energy density of the pulses was restricted to the process window defined in [4], $0.40-0.52 \mathrm{~J} / \mathrm{cm}^{2}$, therefore assuring the best quality of the resulting layer.

After the PLM process, some samples were cut in $0.5 \times 0.5$ $\mathrm{cm}^{2}$ squares in order to characterize them with the van der Pauw set-up. Triangular metal contacts were fabricated on the corners of the samples by a Joule evaporation of an Au:Ge (88:12) $100 \mathrm{~nm}$ layer, and subsequently an e-beam evaporation of an $200 \mathrm{~nm} \mathrm{Au}$ layer without breaking the vacuum.

Photoluminescence (PL) measurements were taken at $10 \mathrm{~K}$ placing the samples inside a Sumitomo Cryogenics $\mathrm{CH}-$ 204SFF cryostat and using the $325 \mathrm{~nm}(\approx 3.8 \mathrm{eV})$ line of a Cd$\mathrm{He}$ laser at $18 \mathrm{~mW}$. The PL emission from the samples,

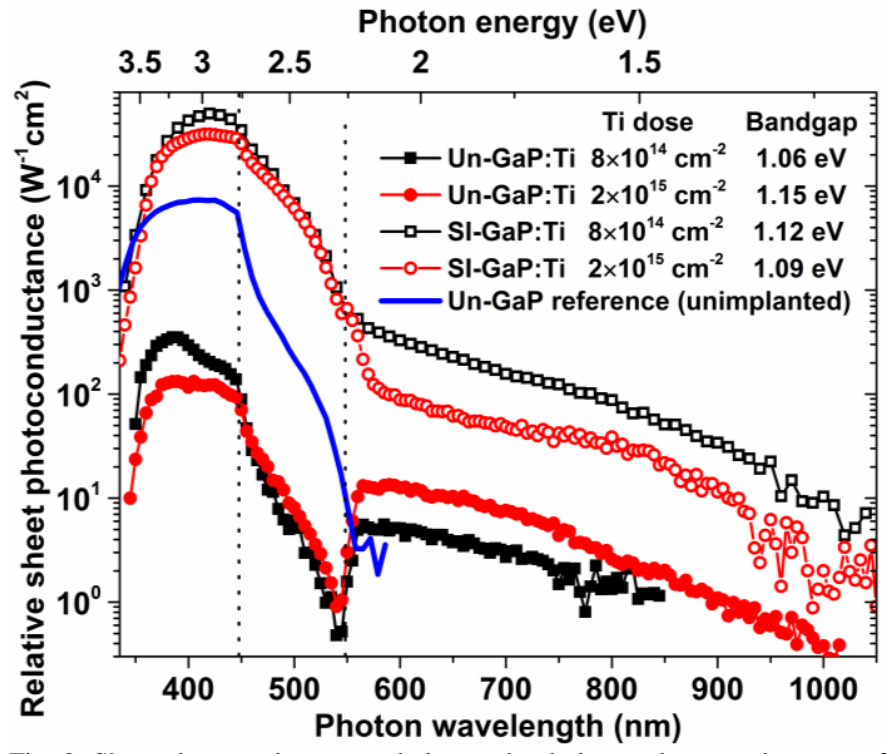

Fig. 2. Sheet photoconductance relative to the darkness sheet conductance of four PLM processed GaP:Ti samples as a function of the photon wavelength, two of them fabricated over Un-GaP substrates (filled symbols), and PLM processed at $0.45 \mathrm{~J} / \mathrm{cm}^{2}$ (low dose sample) and $0.52 \mathrm{~J} / \mathrm{cm}^{2}$ (high dose sample), and the other two fabricated over SI-GaP (hollow symbols), both PLM processed at $0.4 \mathrm{~J} / \mathrm{cm}^{2}$. Black curves are for the lowest Ti dose while red curves are for the highest dose. The dotted vertical lines serve as a reference for the indirect and direct bandgaps of $\mathrm{GaP}$, located at about $550 \mathrm{~nm}(2.26 \mathrm{eV}$, indirect $)$ and $445 \mathrm{~nm}(2.78 \mathrm{eV}$, direct) at room temperature. Also, the spectral relative sheet photoconductance of the unimplanted Un-GaP reference sample PLM processed shown in Fig. 1 is presented in blue. The spectrum of this sample in not shown for wavelengths above $585 \mathrm{~nm}$, where just plain noise is measured, to avoid unreal features produced by the calibration of a plain noise signal.

collected by a high aperture lens, was detected and filtered with a combination of a monochromator (Jobin-Yvon THR 1000), a photomultiplier (Horiba Jobin-Yvon DPM-HV) and a lock-in amplifier.

The van der Pauw set-up was used to conduct two types of measurements at room temperature. First, electronic transport properties of some samples were measured in darkness using a Keithley 4200 SCS equipped with four source and measure units. The Hall effect in this configuration was attempted using a Kepco 50-20MG power source to feed a $0.9 \mathrm{~T}$ electromagnet. Second, the spectral sheet photoconductance of the same samples was characterized. A Keithley 2636 current source was configured to feed $100 \mathrm{nA}$ or $1 \mathrm{~mA}$ between two of the contacts (see sketch in Figure 1), in the case of SI-GaP and $\mathrm{Un}-\mathrm{GaP}$, respectively, while the $\mathrm{AC}$ photogenerated voltage was obtained with a SRS830 DSP lock-in amplifier. A UV-Vis-NIR Horiba iHR320 spectrophotometer with a $250 \mathrm{~W}$ tungsten halogen lamp was used as a light source. A filter wheel with long-pass filters at $320 \mathrm{~nm}, 550 \mathrm{~nm}$ and $750 \mathrm{~nm}$ was placed at the entrance of the monochromator, and slits were configured to obtain a resolution better than $4.62 \mathrm{~nm}$. A chopper was configured to produce AC illumination at $23 \mathrm{~Hz}$. Finally, the spectral power density of the light reaching the sample was measured with a calibrated $\mathrm{Si}$ S2281 Hammamatsu photodiode in photovoltaic mode. 

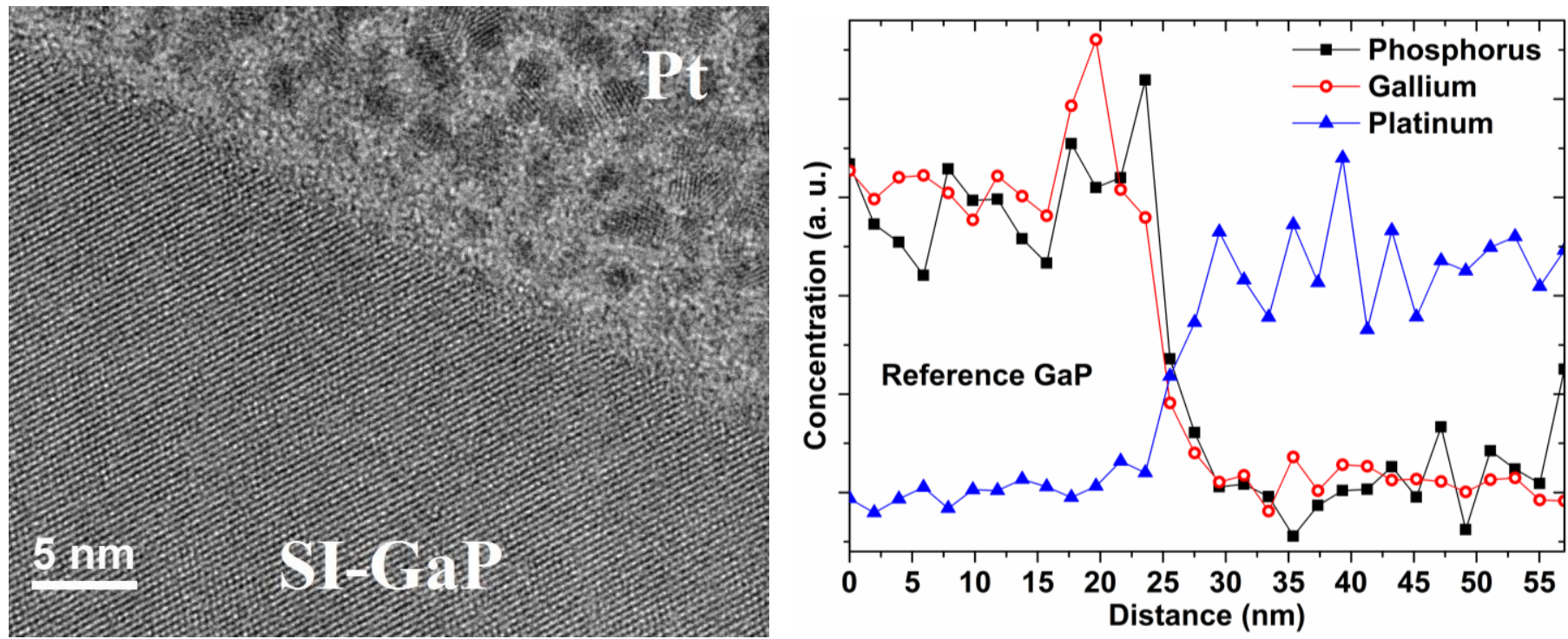

Fig. 3. Left: XTEM image of a reference unimplanted and non-PLM processed SI-GaP sample. Right: XEDS composition profiles of the sample shown in the Left image.

To analyze the crystal quality and composition of the GaP:Ti material by Transmission Electron Microscopy (TEM), lamellas of SI-GaP and SI-GaP:Ti samples were fabricated with dual beam Focused Ion Beam (FIB) scanning electron microscopes, Helios Nanolab 600 and 650 models. To protect the area of interest $(12 \mu \mathrm{m} \times 1.5 \mu \mathrm{m})$, a layer of about $200 \mathrm{~nm}$ of $\mathrm{C}$ rich $\mathrm{Pt}$ was deposited by focused electron beam induced deposition at $5 \mathrm{kV}$ and $1.6 \mathrm{nA}$. Subsequently, a layer of about $500 \mathrm{~nm}$ of $\mathrm{C}$ poorer Pt was deposited by Ga FIB Induced Deposition (FIBID). FIBID always worked at $30 \mathrm{kV}$ and 0.23 nA. For the rough milling of trenches, polishing and undercutting of the lamellas, FIB at $30 \mathrm{kV}$ was used with 2.5 $\mathrm{nA}, 0.23 \mathrm{nA}$ and $2.5 \mathrm{nA}$, respectively. A nanomanipulator was Pt welded to the lamella by FIBID and the lamella was cut by FIB (30 kV $0.23 \mathrm{nA})$ and attached by FIBID to a holder. A final thinning by FIB at $5 \mathrm{kV}$ and $0.68 \mathrm{pA}$ was performed to obtain a lamella of about $5 \mu \mathrm{m} \times 50 \mathrm{~nm}$. Structural properties were analyzed by obtaining the cross-sectional TEM (XTEM) images in a JEOL JEM $3000 \mathrm{~F}$ microscope operating at 300 $\mathrm{kV}$. The scanning mode with a JEOL annular dark field detector with a beam diameter of about $0.7 \mathrm{~nm}$ was used. For semi-qualitative chemical analysis, X-ray Energy Dispersive Spectroscopy (XEDS) was used, extracting the calibrated results through the Oxford Instruments INCA microanalysis suite.

\section{RESULTS}

Figure 1 shows the results of the sheet photoconductance $\left(\Delta \mathrm{G}_{\mathrm{s}}\right)$ and photoluminescence measurements of two unimplanted Un-GaP substrates as a function of the photon energy. The black curves are for as-grown Un-GaP while the red curves are for PLM processed Un-GaP. As it can be seen, the as-grown material has a less intense photoconductance and no measurable photoluminescence, while the PLM processed $\mathrm{GaP}$ shows several PL emission peaks when measuring in the same conditions. The most intense of these PL peaks is close to the energy of the indirect bandgap of $\mathrm{GaP}(2.26 \mathrm{eV}$ at room temperature) and to the beginning of the sheet photoconductance front. No PL peaks were detected for any of the samples at energies below $2 \mathrm{eV}$. No GaP:Ti sample yielded any PL peaks in the measurable limit of the experimental set-up, resulting in spectra similar to the one of the Un-GaP sample without PLM process (black spectrum in Fig. 1).

Figure 2 presents the sheet photoconductance relative to the darkness sheet conductance $\left(\Delta \mathrm{G}_{\mathrm{s}} / \mathrm{G}_{\mathrm{o}}\right.$, see Table 2 for darkness sheet resistance values), of four $\mathrm{GaP}$ samples implanted with $\mathrm{Ti}$ and PLM processed, as a function of the photon wavelength. The relative representation was necessary in order to better compare measurements of samples fabricated with different substrates (Un-GaP and SI-GaP), since the sheet photoconductance curves were many orders of magnitude away. Also, the relative sheet photoconductance response of a reference Un-GaP sample PLM processed is shown for comparative purposes. The SI-GaP reference could not be measured properly due to its extremely high sheet resistance.

The most interesting result shown in this figure is the appearance of photoconductance at wavelengths longer than $550 \mathrm{~nm}$, this is, at energies below the bandgap of bare $\mathrm{GaP}$ $(2.26 \mathrm{eV})$. This feature is not present in the sheet photoconductance spectrum of the Un-GaP reference sample (blue curve in Fig. 2). As it was reported in [4], the origin of this photoconductance would be the high $\mathrm{Ti}$ concentration introduced during the ion implantation and retained after the PLM process. Additionally, a deep collapse of the photoconductance is present for Un-GaP:Ti samples at about $550 \mathrm{~nm}$. As it can be seen in Fig. 2, this collapse has not been measured for samples fabricated over SI-GaP substrates. Finally, Fig. 2 shows also the result of the energy fit of the sheet photoconductance curves at wavelengths above $550 \mathrm{~nm}$ to an indirect bandgap expression as in [4] (depicted as "Bandgap"). These fittings are provided in the Supporting Information section (Fig. S1).

Regarding the sheet resistance obtained from van der Pauw 

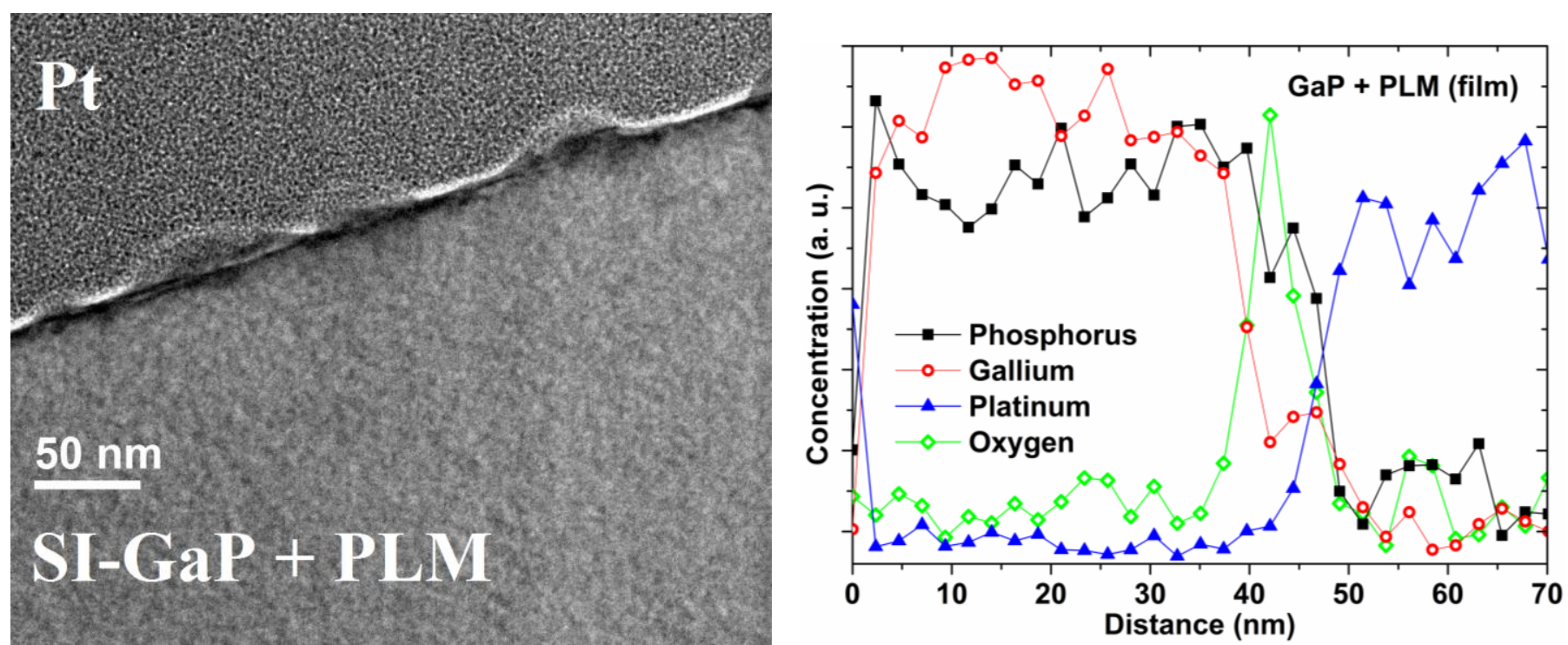

Fig. 4. Left: XTEM image of a reference unimplanted SI-GaP sample PLM processed. Right: XEDS composition profile of a SI-GaP sample PLM processed as the one shown in the Left image. The profile was measured through the new layer present at the GaP/Pt interface.

measurements, values for the samples shown in Figs. 1 and 2 are presented in Table 2. While for Un-GaP samples the values are quite similar, the sheet resistance of samples fabricated on SI-GaP depends strongly on the Ti implantation dose. Furthermore, mobility and carrier concentration of Un$\mathrm{GaP}$ samples present in all cases values in the range of the unimplanted Un-GaP substrate shown in Table 1 (see [4] for temperature dependent curves). On the other hand, Hall effect could not be measured with reliability for SI-GaP:Ti samples, which could be an indication of a very low mobility and/or very high carrier concentration in the GaP:Ti layer.

\begin{tabular}{|c|c|c|}
\hline Type & Ti dose $\left.\mathbf{( c m}^{-2}\right)$ & Sheet resistance $\mathbf{( \Omega / \square ) ~}$ \\
\hline Un-GaP & 0 & 13.8 \\
\hline Un-GaP & $8 \times 10^{14}$ & 20.8 \\
\hline Un-GaP & $2 \times 10^{15}$ & 11.9 \\
\hline SI-GaP & 0 & $0.4-1.1 \times 10^{10}$ \\
\hline SI-GaP & $8 \times 10^{14}$ & $2.1 \times 10^{7}$ \\
\hline SI-GaP & $2 \times 10^{15}$ & $4.7 \times 10^{4}$ \\
\hline
\end{tabular}

Table 2: Room temperature darkness sheet resistance of the samples presented in Figures 1 and 2 (with PLM process). The value of the sheet resistance of the unimplanted SI-GaP substrate was not measured but calculated from the properties of Table 1 given by the supplier.

Figure 3 (Left) shows a XTEM picture of a reference as grown SI-GaP, with no implantation or PLM process. It can be seen how the crystal structure presents no defects, with a very flat transition with the nano-crystalline Pt layer. No native oxide seems to be present in the GaP surface. The composition of this sample through a path crossing the GaP surface can be observed in the Right figure, being the GaP/Pt interface very well defined.

In order to analyze the structural modifications produced by the PLM process on unimplanted GaP, we show in Fig. 4 a XTEM image of an unimplanted SI-GaP sample PLM processed (Left). As it can be seen, there are not extended defects in the GaP substrate when the energy density is inside the window defined in the experimental section. This result is clearly different from the one reported in [17], in which a defective polycrystalline layer was produced when the energy density of the PLM process was very high $\left(0.7 \mathrm{~J} / \mathrm{cm}^{2}\right.$ and 1.2 $\mathrm{J} / \mathrm{cm}^{2}$ ). The high quality of the sample shown in the XTEM image of Fig. 4 Left is also an evidence of the reproducibility of the narrow process window proposed in [4]. However, some features have appeared on the surface. There are some structures like "bubbles", and a new thin layer, shown in white, appears in between these structures. The XEDS composition profile (Fig. 4 Right) of the SI-GaP sample PLM processed shown in the Left part of Fig. 4 has been obtained crossing this new thin surface layer (away from the "bubbles"). Just at the interface a thin O spike is present. No oxygen was measured away from the surface, so we can discard that $\mathrm{O}$ was incorporated during Pt deposition.

Regarding the structure of the "bubble" feature, we present in Fig. 5 a XTEM picture where it is apparent that this feature is also crystalline with the same growth direction of the substrate, probably with some defects (upper left corner of the Left figure). The "bubble" seems to have a lighter color, and the surface boundary can be marked off. Also, in this image, a detail of the new layer seems to indicate that it is mostly amorphous, with a thickness of about $3 \mathrm{~nm}$ except over the "bubble" where it seems to shrink but is still present. A XEDS composition profile was obtained crossing from the substrate to the Pt layer, through a "bubble". The Ga concentration in the "bubble" seems to be higher than the $\mathrm{P}$ concentration. Besides, there seems to be a certain amount of $\mathrm{O}$ in the "bubble". The O profile of the "bubble" would be mixed with the $O$ profile of the amorphous layer, which is clearly seen in the XTEM image but due to its reduced thickness cannot be resolved in the XEDS profile.

The same features ("bubbles" and the surface amorphous layer) are present in the samples implanted with $\mathrm{Ti}$ and this can be observed also in the figures shown in the Supporting Information section. Regarding XEDS profiles, since the Ti concentration is just at the detection limit of the technique, $\mathrm{Ti}$ analysis by XEDS was not reliable. In this sense, previous Time-of-Flight Secondary Ion Mass Spectrometry (ToFSIMS) measurements reported in [4] for similar samples will 

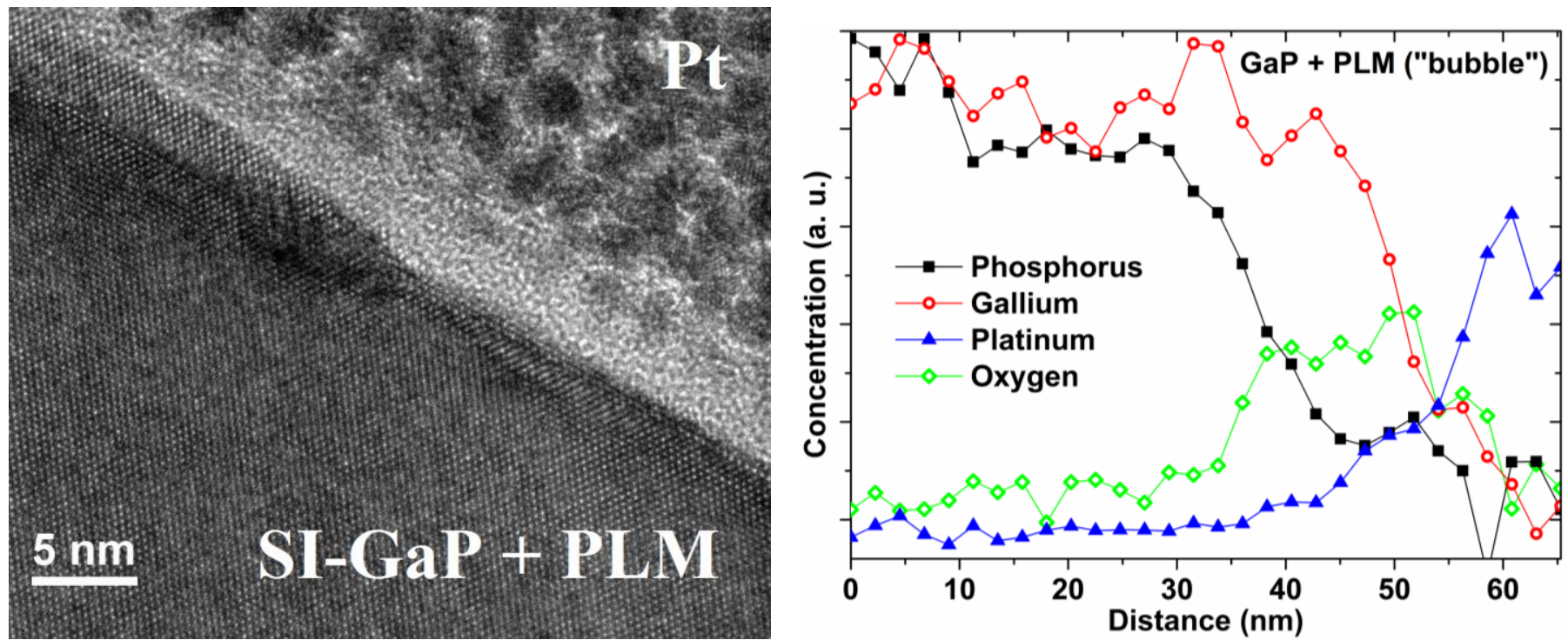

Fig. 5. Left: XTEM image of a reference unimplanted SI-GaP sample PLM processed. Details of a "bubble" feature and the surface amorphous layer are shown. Right: XEDS composition profile of a SI-GaP sample PLM processed as the one shown in the Left image. The profile was measured through one of the "bubbles" present at the GaP/Pt interface.

be used as a reference. Observing the XTEM images of Fig. 6, it can be seen that SI-GaP:Ti samples show some shallow defects with a depth of about $10 \mathrm{~nm}$ that seem to be stacking faults with twins [18]. The sample with the highest Ti dose (Fig. 6 Right) presents a higher defect density along with "volcano" structures inside the twins. At depths beyond approximately $10 \mathrm{~nm}$ the samples present high crystal quality and no defects are detected.

\section{DISCUSSION}

Spectra shown in Fig. 1 indicate that the PLM process induces a more intense photoconductance in Un-GaP with respect to the reference substrate, and extends the photoconductance into the UV. Usually, when an abrupt decrease in the UV range is detected in a photoresponse spectrum it is related to surface recombination [19]. This argument could be reasonable since the sample without PLM process does not have any passivating treatment. The PL spectra shown in the same figure are consistent with this affirmation and add new information: non-radiative recombination seems to be lower in the sample with PLM process. This produces, apart from other less intense peaks, measurable PL at about $2.20 \mathrm{eV}$. This PL peak could be related to radiative recombinations between electrons at the native shallow donor level and holes at an acceptor level at phosphorous sites [20]. Phonon replicas of the $2.20 \mathrm{eV}$ peak at $2.15 \mathrm{eV}$ and $2.11 \mathrm{eV}$ are also visible [21]. No PL peaks were detected in the unimplanted not PLM processed sample, nor in the Ti implanted and PLM processed samples (not shown in Fig. 1).

In Fig. 2 the above bandgap sheet photoconductance of GaP:Ti samples has the same shape as the one of the unimplanted PLM processed GaP sample, this is, it is not reduced abruptly in the UV at about $2.75 \mathrm{eV}(\approx 450 \mathrm{~nm})$ as in the spectrum of the non PLM processed Un-GaP sample (Fig. 1). Moreover, in Fig. 2 we can still detect the indirect and direct bandgap fronts at about $2.26 \mathrm{eV}(\approx 550 \mathrm{~nm})$ and 2.78 $\mathrm{eV}(\approx 445 \mathrm{~nm})$ for all the GaP:Ti samples. Because of all these evidences, we propose that the PLM process passivates the GaP surface.

As it can be seen in Figs. 4-6, the PLM process produces a new amorphous thin layer of about $3 \mathrm{~nm}$ that seems to be composed of Ga, $\mathrm{P}$ and $\mathrm{O}$ (Fig. 4 Right). We propose that this new layer passivates the surface of the GaP based materials (both with and without $\mathrm{Ti}$ implantation) and reduces the surface recombination velocity. The fact that no PL peaks were measured for GaP:Ti samples would point that, although the amorphous layer created by the PLM process would still be passivating these samples, shallow structural defects (stacking faults, see Fig. 6) might be acting as non-radiative recombination centers. Also, Ti levels in the GaP:Ti material could be promoting non-radiative transitions, especially in the implantation tail region. In the tail region the Ti concentration would be low and Ti levels would be acting as recombination centers instead of as an IB [6]. This effects will have to be taken into account in any attempt to make a viable photovoltaic device. Regarding the bare GaP sample without PLM process, since not even a bad quality native oxide has been observed (see Fig. 3 Left), non-radiative recombination at the surface would be high enough as to affect both PL and sheet photoconductance measurements.

Regarding the composition of the passivating layer, although the XEDS technique is not able to give a completely reliable $\mathrm{Ga} / \mathrm{P}$ ratio, the change from a higher than one ratio in all substrate characterizations to a lower than one ratio in the thin surface layer (Fig. 4 Right), would point to a composition of this layer with more $\mathrm{P}$ than Ga. Another indication of this substoichiometric composition was obtained from a new analysis of the ToF-SIMS data used for [4], confirming the XEDS result (see Fig. S4 in the Supporting Information section). More analysis is underway in order to confirm the composition of this layer. GaP passivation by surface suboxides has been previously reported for Schottky diodes 

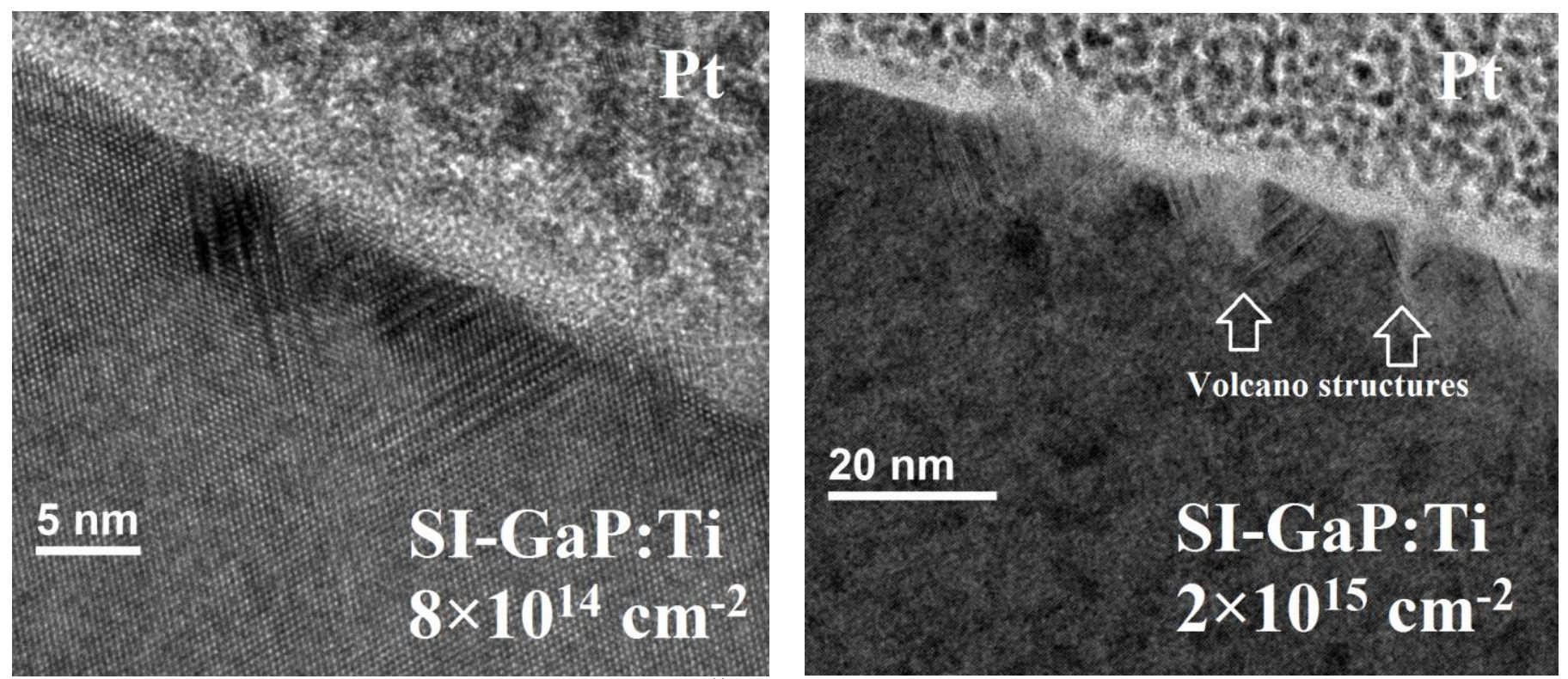

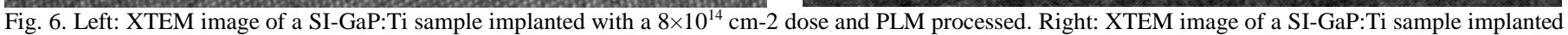
with a $2 \times 10^{15} \mathrm{~cm}-2$ dose and PLM processed.

[22], eliminating the sub-bandgap photoresponse generated at the surface states, and for MOS devices [23], reducing the $\mathrm{GaP}$ surface states. The passivation of the surface by this layer would be coherent also with the fact that the sheet photoconductance of $\mathrm{GaP}: \mathrm{Ti}$ measured at photon energies below the bandgap would be originated in the $\mathrm{Ti}$ implanted and PLM processed layer. If the origin of this below bandgap photoconductance were the surface states, it would had been present in the unimplanted and not PLM processed GaP sample (Fig. 1). This fact would discard the oxygen impurities that could be introduced by the surface during the PLM process as the origin of the below bandgap photoconductivity, since the PLM unimplanted sample did not show any below bandgap activity.

The PLM consists of a fast melting process followed by a fast recrystallization that pushes the impurities towards the surface, expelling part of the implanted dose. If the impurity concentration is very high, the recrystallization process can be unstable [24]. In this sense, there is an impurity concentration limit that can be experimentally attained resulting in a supersaturated layer with high crystal quality [25]. Comparing the depth of the defects shown in Fig. 6 with the $\mathrm{Ti}$ concentration profile after PLM of similar samples shown in Ref. [4], it seems that Ti concentrations as high as $10^{20} \mathrm{~cm}^{-3}$ can be obtained without any defect. In general, the solid solubility limit of deep levels in III-V semiconductors is around $10^{17} \mathrm{~cm}^{-3}$ [26]. Therefore, the combination of ion implantation and PLM, as non-equilibrium techniques, seems to be adequate to fabricate supersaturated GaP:Ti. On the other hand, the volcano-like structures shown in Fig. 6 Right are typical fingerprints of a defect known as cellularbreakdown [24], which consists of a tubular structure with an accumulation of the impurity in the tube walls, and is undesirable because of the inhomogeneous distribution of the impurity. However, more structural analysis is needed in order to confirm the nature of these defects [27].
More information about the optoelectronic properties of GaP:Ti can be obtained by comparing the sheet photoconductance spectra shown in Fig. 2. Some similarities between Ti-implanted samples fabricated on Un-GaP and SI$\mathrm{GaP}$ can be inferred. First, the presence of an intense subbandgap photoconductance, with an energy front having approximately the same energy. This would indicate that the GaP:Ti layer is essentially the same from the optoelectronic properties point of view, regardless of the substrate doping. As pointed in [4], the optimum position would be $0.85 \mathrm{eV}$ from the IB to the $\mathrm{CB}$ (or the $\mathrm{VB}$ ), or its complementary to the $\mathrm{GaP}$ bandgap, $1.41 \mathrm{eV}$ approximately. The energy position obtained here is similar to the ones reported previously for localized $\mathrm{Ti}$ deep donor levels in GaP, i.e. GaP:Ti with Ti concentration below the solid solubility limit, which would be at about $1 \mathrm{eV}$ [28][29], $1.05 \mathrm{eV}$ [30] or $1.15 \mathrm{eV}$ [31].

Second, the passivating effect of the PLM is also similar on both substrate types: there is not an abrupt decay at about 450 $\mathrm{nm}(2.75 \mathrm{eV})$ as in the spectrum of the sample not PLM processed (Fig. 1). Also, both the indirect $(2.26 \mathrm{eV})$ and direct $(2.78 \mathrm{eV})$ bandgaps of $\mathrm{GaP}$ can be detected.

However, there is a crucial difference. The abrupt collapse at $550 \mathrm{~nm}$ shown for Un-GaP:Ti samples is not present in SIGaP:Ti samples. A first possible explanation for the collapse feature could be a strong recombination in the GaP:Ti layer due to a high absorption coefficient value at about $550 \mathrm{~nm}$. A similar feature occurs for all the spectra at $350 \mathrm{~nm}$. In fact, the abruptness of the collapse is similar. In theory, when the absorption coefficient is very high, the accumulation of photogenerated carriers in a very thin surface layer could produce a strong decrease of the lifetime, and therefore an abrupt reduction of the photoresponse. When the wavelength of the photon is below $550 \mathrm{~nm}$, the thicker substrate adds its sheet photoconductance and the response increases. Regarding the spectra corresponding to the SI-GaP samples, the semiinsulator character of the substrate would make it more 
sensitive and thus its photoconductance is much stronger at energies above the bandgap. In this sense, the substrate contribution would hide the collapse of the sheet photoconductance in the GaP:Ti layer.

Although the scenario described in the previous paragraph could make sense, oddly, the collapse begins just below the energy of the bandgap of $\mathrm{GaP}$ (around $565 \mathrm{~nm}, 2.19 \mathrm{eV}$ ), and this coincidence suggests that there might be another origin for this phenomenon. Similar spectra have been found in [32][33][34]. In those works the collapse found at the bandgap energy was attributed to the occurrence of negative photoconductivity at energies below the bandgap. Obviously, if the photoconductivity is positive at energies above the bandgap, just at the bandgap energy the resulting photoconductivity must pass through zero, and that would be the collapse shown in Fig. 2 at $540 \mathrm{~nm}$. The origin of this negative photoconductivity has been described thoroughly in the literature [35] and usually several deep and/or shallow levels are involved in this phenomenon. Fig. 7 shows a band diagram corresponding to a hypothesized model to describe the sheet photoconductance collapse produced by negative photoconductivity processes. First, process 1 generates one hole in the VB while one electron is trapped in a shallow level. If this electron is not emitted to the $\mathrm{CB}$ fast enough, there is a certain probability of producing an electron-hole recombination in a deep level (process 2). Finally, the electron in the shallow level would be emitted to the CB (process 3), reaching equilibrium. The time between process 2 and 3 would account for negative photoconductivity, since there would be less carriers than in darkness condition. In the $\mathrm{GaP}: \mathrm{Ti}$ case, shallow levels from the original GaP dopant, and shallow and deep levels from $\mathrm{Ti}$, could be responsible for these processes.

The attribution of the photoconductance collapse to a switch from negative to positive photoconductivity is supported by an abrupt change of the measured phase at the wavelength of the collapse, as shown in the supporting material.

The magnitude of negative photoconductivity might depends also on several factors such as light intensity, polarization [34], temperature [36] or even the chopping frequency of the set-up [35]. Besides, the measurement of negative photoconductivity can be the result of having both phenomena, negative and positive, at the same time, dominating the negative due to the experimental conditions. In fact, Un-GaP:Ti samples showing negative photoconductivity (Fig. 2) illuminated with the monochromator and measured with the lock-in set up described previously (maximum light power density of $5 \times 10^{-5} \mathrm{~W} / \mathrm{cm}^{2}$ ), showed clearly positive photoconductivity at energies below the $\mathrm{GaP}$ bandgap when measured with a red LED $(650 \mathrm{~nm})$ as light source.

Regarding the SI-GaP:Ti samples, only positive photoconductivity is measured in the whole wavelength range. Our hypothesis is that the high concentration of shallow levels together with the Ti impurities in the Un-GaP substrate are playing an essential role in the processes described in Fig. 7. Since the SI-GaP substrate has a much lower density of shallow levels, positive photoconductivity would dominate,

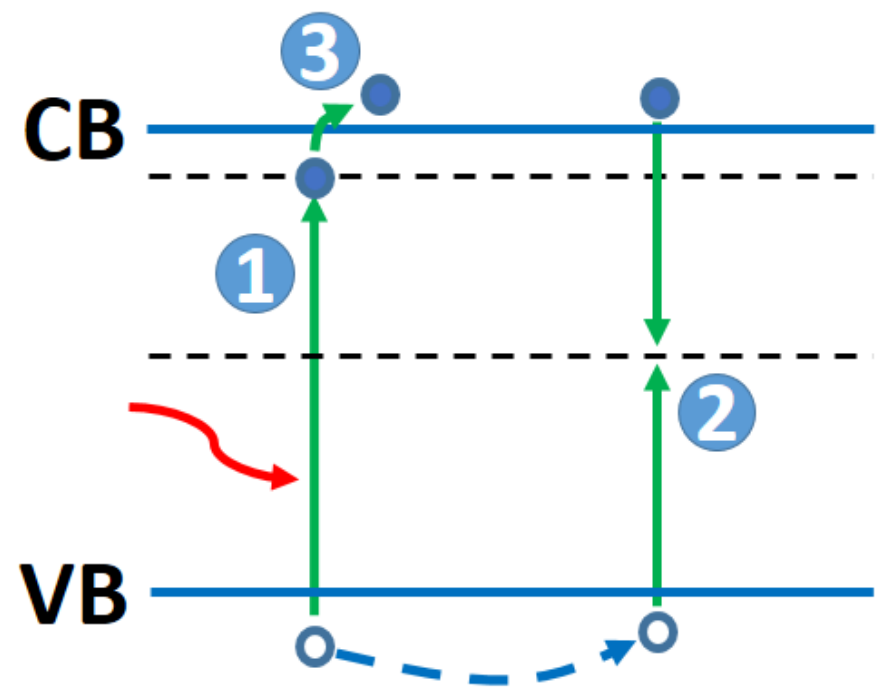

Fig. 7. Band diagram model used to qualitatively explain the sheet photoconductance collapse shown in Fig. 2.

precluding the appearance of the collapse. More work is underway to better understand the negative and positive photoconductivity effects in these materials.

To finish with the electronic transport properties of the GaP:Ti material, the room temperature sheet resistance measurements are shown in Table 2. For the SI-GaP:Ti samples, given the extremely high sheet resistance of the substrate, the measured sheet resistance would be due just to the GaP:Ti surface layer. It is striking how for just an increase of roughly twofold in the dose, the sheet resistance decreases more than two orders of magnitude. This dependence could be related to some kind of insulator-to-metal transition but since the Hall effect could not be measured, no definitive conclusions can be obtained. However, if GaP:Ti surface layers fabricated over Un-GaP and SI-GaP are similar, as quoted above, the high sheet resistance measured for SIGaP:Ti samples (above $10^{4} \Omega / \square$ ) would not affect the measurement of the samples fabricated over Un-GaP substrates. In this case the substrate would dominate the parallel conduction, in agreement with values shown in Table 2.

\section{CONCLUSIONS}

We have confirmed the reliability of the PLM process window obtained in previous studies to recrystallize SI-GaP implanted with high Ti doses. GaP:Ti materials exhibit high sheet photoconductance at energies below the $\mathrm{GaP}$ bandgap independently of the substrate conductivity, and this could enhance the quantum efficiency of a photovoltaic device fabricated with this material. We also found out the presence of a new amorphous layer composed of $\mathrm{Ga}, \mathrm{P}$ and $\mathrm{O}$, originated during the PLM process, that could be effectively passivating the surface of $\mathrm{GaP}$ and $\mathrm{GaP}: \mathrm{Ti}$ samples. This surface modification could be critical for IB materials based on III-V semiconductors, since the PLM would recrystallize and passivate in the same process, being this result of interest for high efficiency solar cells. This passivation could also raise the efficiency of bare $\mathrm{GaP}$ devices, which usually is very low due to the deficient state-of-the art passivation techniques. 
We studied the structural quality of $\mathrm{GaP}: \mathrm{Ti}$ materials fabricated with a high Ti implantation dose concluding that the quality is good and the layer is monocrystalline but some shallow defects still exist. Finally, two hypotheses were proposed to explain the deep collapse at the energy of the bandgap of GaP that was found on GaP:Ti samples fabricated on undoped GaP substrate with high conductivity. The first hypothesis is related to surface recombination while the second is related to negative photoconductivity.

\section{ACKNOWLEDGMENT}

Authors would like to acknowledge the C.A.I. de Técnicas Físicas of the Universidad Complutense de Madrid for ion implantation and evaporation processes. Also, the Institute for Optoelectronics Systems and Microtechnology of the Universidad Politécnica de Madrid for photoluminescence experiments. The fabrication of TEM samples were conducted in the Laboratorio de Microscopias Avanzadas at the Instituto de Nanociencia de Aragon (LMA-INA) of the Universidad de Zaragoza. Authors acknowledge the LMA-INA for offering access to their instruments and expertise, and the Centro Nacional de Microelectrónica at the Moncloa Campus for the TEM and XEDS measurements.

This work was partially supported by the Spanish Ministry of Science, Innovation and Universities under grants TEC2017-84378-R and RTI 2018-096498-B-I00. The work of D. Montero was supported by the Spanish MINECO under contract BES-2014-067585. This work is part of the project MADRID-PV2 P-2018/EMT-4308 funded by the Regional Government of Madrid with the support from FEDER funds.

\section{REFERENCES}

[1] T. J. Grassman, J. A. Carlin, B. Galiana, et al., "MOCVDgrown $\mathrm{GaP} / \mathrm{Si}$ subcells for integrated III-V/Si multijunction photovoltaics," IEEE Journal of Photovoltaics, vol. 4, pp. 972-980, March 2014, 10.1109/JPHOTOV.2014.2308727.

[2] C. Zhang, N. N. Faleev, L. Ding, et al., "Hetero-emitter $\mathrm{GaP} / \mathrm{Si}$ solar cells with high Si bulk lifetime," presented at the IEEE $43^{\text {rd }}$ Photovoltaic Specialists Conference, Portland, OR, USA, Jun. 5 - 10, 2016, pp. 1950-1953.

[3] X. Lu, S. R. Huang, M. Diaz, R. L. Opila and A. Barnett, "Wide band gap Gallium Phosphide solar cells for multijunction solar cell system," presented at the 35th IEEE Photovoltaic Specialists Conference, Honolulu, HI, USA, June 20 - 25, 2010, pp. 002079-002083.

[4] J. Olea, A. del Prado, E. García-Hemme, et al., "Strong subbandgap photoconductivity in GaP implanted with Ti," Prog. Photovolt. Res. Appl., vol. 26, pp. $214-222$, Dec. 2017, 10.1002/pip.2974.

[5] A. Luque and A. Marti, "Increasing the efficiency of ideal solar cells by photon induced transitions at intermediate levels," Phys. Rev. Let., vol. 78, pp. 5014 - 5017, June 1997, 10.1103/PhysRevLett.78.5014.

[6] A. Luque, A. Martí, E. Antolin and C. Tablero, "Intermediate band versus levels in non-radiative recombination," Physica B, vol. 382, pp. 320-327, June 2006, 10.1016/j.physb.2006.03.006.

[7] A. Luque and A. Martí, "A metallic intermediate band high efficiency solar cell," Prog. Photovolt. Res. Appl., vol. 9, pp. 73 - 86, April 2001, 10.1002/pip.354.

[8] H. S. Saini, M. K. Kashyap, M. Kumar et al., "Generating magnetic response and half-metallicity in $\mathrm{GaP}$ via dilute Ti-doping for spintronic applications," Journal of Alloys and Compounds, vol. 649, pp. 184-189, 2015.

[9] P. Palacios, J. J. Fernandez, K. Sanchez, J. C. Conesa and P. Wahnon, "First-principles investigation of isolated band formation in half-metallic TixGa1-xP ( $\mathrm{x}=0.3125-$ 0.25)," Phys. Rev. B, vol. 73, Art. no. 085206, Feb. 2006, 10.1103/PhysRevB.73.085206.

[10]C. Tablero, "Optoelectronic properties analysis of $\mathrm{Ti}$ substituted GaP," J. Chem. Phys., vol. 123, Art. no. 184703, Nov. 2005, 10.1063/1.2107367.

[11]Z. Werner, M. Barlak, R. Ratajczak, P. Konarski, A. M. Markov and R. Heller, "Electron-beam pulse annealed Tiimplanted GaP," J. Appl. Phys., vol. 120, Art. no. 085103, August 2016, 10.1063/1.4961518.

[12] K. M. Yu, W. Walukiewicz, J. W. Ager III et al., "Multiband GaNAsP quaternary alloys," Appl. Phys. Lett. Vol. 88, Art. no. 092110, March 2006, 10.1063/1.2181627.

[13] M. A. Scarpulla, U. Daud, K. M. Yu et al., "Diluted magnetic semiconductors formed by ion implantation and pulsed-laser melting," Physica B, vol. 340 - 342, pp. 908 - 912, Dec. 2003, 10.1016/j.physb.2003.09.113.

[14] M. A. Scarpulla, B. L. Cardozo, R. Farshchi et al., "Ferromagnetism in $\mathrm{Ga}_{1-\mathrm{x}} \mathrm{Mn}_{\mathrm{x}} \mathrm{P}$ : evidence for inter-Mn exchange mediated by localized holes within a detached impurity band," Phys. Rev. Lett., vol. 95, Art. no. 207204, 2005.

[15] C. R. Allen, J. M Woodall and J. H. Jeon, "Results of a gallium phosphide photovoltaic junction with an AR coating under concentration of natural sunlight," Sol. Energy. Mater. Sol. Cells., vol. 95, pp. 2655-2658, Sept. 2011, 10.1016/j.solmat.2011.05.034.

[16]S. Ruhle, "Tabulated values of the Shockley-Queisser limit for single junction solar cells," Solar Energy, vol. 130, pp. 139-147, June 2016, 10.1016/j.solener.2016.02.015.

[17]D. Pastor, J. Olea, M. Toledano-Luque, et al., "Laser thermal annealing effects on single crystal gallium phosphide," J. Appl. Phys., vol. 106, Art. no. 053510, Sept. 2009, 10.1063/1.3187902.

[18]M. A. Scarpulla, "III-Mn-V ferromagnetic semiconductors synthetized by ion implantation and pulsed-laser melting," Ph.D. dissertation, Univ. of Cal., Berkeley, Cal., 2006, p. 145.

[19] X. Lu, R. Hao, M. Diaz, et al., "Improving GaP solar cell performance by passivating the surface using $\mathrm{Al}_{\mathrm{x}} \mathrm{Ga}_{1-\mathrm{x}} \mathrm{P}$ epi-layer," IEEE J. Electron Devices Society, vol. 1, pp. 111-116, May 2013, 10.1109/JEDS.2013.2266410.

[20]L. Samuleson, P. Omling and H. G. Grimmeiss, "Electrical and optical properties of $\mathrm{GaP}$ grown on $\mathrm{Si}$ by MOVPE," J. Crystal Growth, vol. 68, pp. 340-344, Sept. 1984, 10.1016/0022-0248(84)90435-4. 
[21]T. K. Sharma, V. K. Dixit, T. Ganguli, et al., "Optimization of the properties of MOVPE-grown GaP epitaxial layers on GaP (111)B substrates," Semicond. Sci. Technol., vol. 23, Art. no. 075031, May 2008, 10.1088/0268-1242/23/7/075031.

[22] M. Somogyi, "Investigation of oxidized $\mathrm{A}^{\mathrm{III}} \mathrm{B}^{\mathrm{V}}$ surfaces by photoresponse," Acta Physica Academiae Scientiarum Hungaricae, vol. 48, pp. 153 - 160, 1980.

[23] T. Ikoma and H. Yokomizo, "C-V characteristics of GaP MOS diode with anodic oxide film," IEEE Transaction on Electron Devices, vol. 23, pp. 521 - 523, May 1976, 10.1109/T-ED.1976.18441

[24] A. J. Akey, D. Recht , J. S. Williams et al., "Single-phase filamentary cellular breakdown via laser-induced solute segregation”, Adv. Funct. Mater., vol. 25, pp. 4642-4649, June 2015, 10.1002/adfm.201501450.

[25] W. Yang, A. J. Akey, L. A. Smillie et al., "Au-rich filamentary behavior and associated subband gap optical absorption in hyperdoped Si," Phys. Rev. Mat., vol. 1, Art. no. 074602, Dec. 2017, 10.1103/PhysRevMaterials.1.074602

[26]B. Clerjaud, "Transition-metal impurities in III-V compounds," Journal of Physics C-Solid State Physics, vol. 18, pp. 3615-3661, 1985, 10.1088/00223719/18/19/005

[27] A. J. Akey, D. Recht, J. S. Williams et al., "Single-phase filamentary cellular breakdown via laser-induced solute segregation," Adv. Func. Mat., vol. 25, pp. 4642 - 4649, June 2015, 10.1002/adfm.201501450.

[28] C. D. Brandt, A. M. Hennel, T. Bryskiewicz, K. Y. Ko, L. M. Pawlosicz and H. C. Gatos, "Electronic and optical properties of $\mathrm{Ti}$ doped $\mathrm{GaAs}$ and InP; semiinsulating InP," J. of Appl. Phys., vol. 65, pp. 3459 - 3469, June 1989, 10.1063/1.342614.

[29] P. Roura, G. Bremond, A. Nouailhat and G. Guillot, "Identification of the titanium-related levels in GaP," Appl. Phys. Lett., vol. 51, pp. 1969 - 1698, 1987, 10.1063/1.98547.

[30] W. Ulrici, K. Friedland, L. Eaves et al., "Optical and electrical studies of GaP:Ti," Phys. Stat. Sol. (b), vol 150, pp. 177 - 190, Nov. 1988, 10.1002/pssb.2221500121.
[31]G. Bremond, G. Guillot, P. Roura and W. Ulrici, "Identification of the Ti donor level in GaP." Semicond. Sci. Technol., vol 6, pp. 85-88, 1991.

[32]J. R. Barret and G. C. Gerhard, "Negative photoconductivity in gold-doped silicon," J. of Appl. Phys., vol. 38, pp. 900, 1967, 10.1063/1.1709448.

[33]E. M. Godzhaev, A. M. Nazarov, Kh. O. Sadygova and Kh. S. Khalilova, "Negative photoconductivity in TlIn ${ }_{x} \mathrm{Nd}_{\mathrm{x}} \mathrm{Se}_{2}(0<\mathrm{x}<0.05)$ solid solutions," Inorganic Materials, vol. 37, pp. 995, Oct. 2001, 10.1023/A:101236260.

[34] G. C. Seong, N. Tschang-Uh and K. K. Eun, "Deep levels states and negative photoconductivity in $\mathrm{n}-\mathrm{ZnO} / \mathrm{p}-\mathrm{Si}$ hetero-junctions diodes," Current Applied Physics, vol. 14, pp. 223, March 2014, 10.1016/j.cap.2013.11.026

[35] C. M. Penchina, J. S. Moore and N. Jr. Holonyak, "Energy levels and negative photoconductivity in cobaltdoped silicon," Phys. Rev., vol. 143, pp. 634, March 1966, 10.1103/PhysRev.143.634

[36] K. Hye Ryong, K. Sung, K. Chang Oh and C. Suk-Ho, "Temperature-dependent negative photoconductivity of undoped ZnO films," Thin solid films, vol. 518, pp. 305, Nov. 2009, 10.1016/j.tsf.2009.06.049. 